\title{
PERSPECTIVE \\ Social networking and mental health: looking beyond frequency of use and towards mechanisms of action
}

\author{
Kiara R. Timpano (D) and Courtney Beard ${ }^{2}$ \\ Neuropsychopharmacology (2020) 45:905-906; https://doi.org/10.1038/s41386-020-0629-8
}

Social networking sites have become ubiquitous in daily life. A recent estimate of monthly active Facebook users alone is over 2.4 billion. While social media platforms can be used to seek peer support and may provide a greater sense of community, social media use has increasingly also been evoked as a possible explanation for the dramatic rise in mental illness and suicide observed in younger cohorts [1]. In line with this notion, studies examining the association between social networking site usage, mental health outcomes (e.g., depression, anxiety, loneliness), and problematic health behaviors (e.g., substance use) have grown exponentially over the past few years (for a meta-analysis, see [2]). Yet despite strong hypotheses, actual effect size estimates of the association between social media use and mental health symptoms are small in magnitude and unreliable [3].

One key explanation for these mixed findings lies in the inconsistent ways studies operationalize social media use. Often the focus is simply on frequency or duration of use rather than types of, or motivations for use. For example, whereas passive use (e.g., passively viewing) may be associated with worse psychiatric symptoms, other types of use (e.g., posting or commenting) may be associated with improved well-being and civic engagement [2]. Research on this topic is further limited by a lack of prospective and experimental studies. While social media usage may increase depression and anxiety symptoms, it is equally plausible that worsening depression or anxiety leads to an increase in passive social media use. Longitudinal research is furthermore lacking that could help clarify potential developmental or cohort differences in causality; associations between types of use and mental health outcomes may differ between older and younger adults using social networking sites [1].

The preponderance of cross-sectional studies presents a particular challenge in identifying potential mechanisms linking social media use and mental health symptoms. In one of the few experimental studies, students who took a 1-week break from Facebook experienced improved well-being and increases in positive mood, compared with those with continued Facebook usage. These effects were especially pronounced for those with heavy or passive usage, and those who endorsed envy while viewing posts [4]. What remains less clear is exactly how passive or heavy use contributes to increased negative mood, and whether that effect is specific. The lack of studies focused on mechanisms consequently means that the field lacks a comprehensive theoretical model to explain the current mixed findings.

Given the exponential growth of social networking sites and preliminary evidence that some forms of social media use may cause or worsen psychopathology, a strategic program of research moving forward is needed. First, future studies should go beyond merely assessing frequency or duration of social media use and instead more carefully characterize basic types of use, motivations for use (e.g., loneliness), or identify different patterns of engagement with social networking sites. Objective measures of use should be prioritized over self-report. Technological software advances (e.g., passive data collection apps and algorithms) will aid in more accurate and less biased measures of use, and similarly, advances in machine learning and natural language processing will further help researchers catalyze their investigations on the link between social media use and mental health outcomes.

A second call to action for the field is to increase the number of experimental studies that test potential mechanisms. To date, only a handful of studies have considered potential factors that could help explain an association between worse mental health outcomes and use of social networking sites. One notable exception is cross-sectional research focused on "fear of missing out" (FOMO), with results indicating FOMO may mediate the relationship between more frequent and problematic smartphone use and anxiety, specifically [5]. However, no experimental studies have examined this mediational model, raising questions about the proposed causality. There are furthermore numerous additional pathways that have only received marginal attention. For example, social media use may (or may not) have a direct impact on declines in face-to-face social interactions, which when combined with more passive use may impact levels of belongingness and subsequent downstream effects on mood. Social media use could also contribute to increased reliance on maladaptive coping strategies, such as distraction, avoidance, and suppression, while simultaneously hindering the use of more adaptive behaviors. A range of cognitive (e.g., self-efficacy), behavioral (e.g., substance use), and emotional (e.g., loneliness, sustained negative affect) factors could act as additional mediators of these relationships. The impact on or moderating role of executive function, including attention and decision making, may further present a promising line of inquiry. Research should also examine the specific effects of social comparison and social shaming, particularly with respect to gender differences, and how they may lay a foundation for cyber bullying (see [6] for a review). Health behaviors, such as increased sedentariness, interrupted sleep, and substance use should also be investigated, along with differential effects across gender. In addition to individual difference factors, researchers may also want to consider more group-level vulnerabilities, including the sharing of misinformation, which could have direct impacts on increasing 1Department of Psychology, University of Miami, 5665 Ponce de Leon Blvd, Coral Gables, FL, USA and ${ }^{2}$ McLean Hospital and Harvard Medical School, Belmont, MA, USA
Correspondence: Kiara R. Timpano (kiaratimpano@gmail.com)

Received: 18 November 2019 Revised: 20 January 2020 Accepted: 24 January 2020

Published online: 7 February 2020 
stigma, and may also provide greater access to information about ways to inflict self-harm.

Studies addressing these critical questions are needed to better understand not only the degree of the link between social media use and mental health outcomes, but to help formulate a comprehensive model of mechanisms underlying this association. This type of work is vital to empowering intervention development. Currently, the treatment and prevention literature for problematic social media use is in its infancy, with extant studies designed to target more narrow problem behaviors, such as internet or gaming addiction. Interventions have ranged from school-based, media-literacy programs for adolescents and their parents, to app-based, individual-level treatments. Preliminary data indicate that psychoeducation, cognitive, and behavioral strategies (e.g., self and app-based monitoring, limit-setting, identification of alternative activities), and motivational interviewing approaches may all be helpful to help reduce frequency of gaming or internet use [7].

Since social networking sites are now inextricably woven into our social fabric, future solutions focused on reducing use will not be the only ones to consider. Instead, interventions focused on changing or shifting the nature of social media use will be necessary, which could highlight and capitalize on the positive aspects of social media. The needed experimental and mediational studies will likely inform subsequent interventions directly, by exploring the myriad of mechanisms that could underlie the emergence of problematic social media use and associated negative outcomes. It is likely that personalized intervention approaches-along the lines of precision medicine-will provide the best approach to reducing the negative ramifications of problematic internet use. Until such specific approaches are developed, social media usage should be assessed similar to the traditional assessment of social support or substance use. Clinicians may need to work with patients to identify the idiographic benefits they derive from social media use, while simultaneously minimizing hurtful effects.
FUNDING AND DISCLOSURE

$\mathrm{CB}$ and KRT have received funding from $\mathrm{NIMH}$ and various foundations. $C B$ and KRT declare no potential conflict of interest.

\section{AUTHOR CONTRIBUTIONS}

Both authors made substantial contributions to the conception and drafting of the work. KRT provided the initial review of the literature, and both authors collaborated on the initial outline of the commentary. CB wrote a first draft of the first section of the review, while KRT wrote a first draft of the second section of the review. Both authors revised the draft and have approved the final version for publication.

\section{ADDITIONAL INFORMATION}

Publisher's note Springer Nature remains neutral with regard to jurisdictional claims in published maps and institutional affiliations.

\section{REFERENCES}

1. Twenge JM, Cooper AB, Joiner TE, Duffy ME, Binau SG. Age, period, and cohort trends in mood disorder indicators and suicide-related outcomes in a nationally representative dataset, 2005-2017. J Abnorm Psychol. 2019;128:185-99.

2. Yoon S, Kleinman M, Mertz J, Brannick M. Is social network site usage related to depression? A meta-analysis of Facebook-depression relations. J Affect Disord. 2019;248:65-72.

3. Seabrook EM, Kern ML, Rickard NS. Social networking sites, depression, and anxiety: a systematic review. JMIR Ment Health. 2016;3:e50.

4. Tromholt M. The Facebook experiment: quitting Facebook leads to higher levels of well-being. Cyberpsychology Behav Soc Netw. 2016;19:661-6.

5. Elhai JD, Yang H, Fang J, Bai X, Hall BJ. Depression and anxiety symptoms are related to problematic smartphone use severity in Chinese young adults: fear of missing out as a mediator. Addict Behav. 2020;101:105962.

6. Ansary NS. Cyberbullying: concepts, theories, and correlates informing evidencebased best practices for prevention. Aggress Violent Behav. 2019;50:101343.

7. King DL, Delfabbro PH, Doh YY, Wu AM, Kuss DJ, Pallesen S, et al. Policy and prevention approaches for disordered and hazardous gaming and Internet use: an international perspective. Prev Sci. 2018;19:233-49. 